Supporting Information

\title{
Relation between Overall Rate of ATRP and Rates of Activation of Dormant Species
}

\author{
Pawel Krys ${ }^{\dagger}$, Thomas G. Ribelli ${ }^{\dagger}$, Krzysztof Matyjaszewski ${ }^{\dagger, *}$, Armando Gennaro ${ }^{\ddagger}$,
}

${ }^{\dagger}$ Center for Macromolecular Engineering, Department of Chemistry, Carnegie Mellon University, 4400 Fifth Avenue, Pittsburgh, Pennsylvania 15213

*Department of Chemical Sciences, University of Padova, Via Marzolo 1, 35131 Padova, Italy

Corresponding authors.

*(K.M.) E-mail: km3b@andrew.cmu.edu

*(A.G.) E-mail: armando.gennaro@unipd.it 
Kinetic models and rate coefficients for PREDICI simulations

In all simulations apparent values of termination rate coefficients were used, with no chain length dependence. The chosen values are consistent with typical termination rate coefficients observed for small molecules $\left(k_{\mathrm{t} 0}\right)$ and macromolecules $\left(k_{\mathrm{tR}}, k_{\mathrm{tc}}, k_{\mathrm{td}}\right)$.

Table S1. Kinetic model and rate coefficients for modeling of normal ATRP of methyl acrylate, under conditions: [MA]:[MBP]:[Cu $\left.{ }^{\mathrm{I}} \mathrm{Br}\right]:\left[\mathrm{Cu}^{\mathrm{II}} \mathrm{Br}_{2}\right]:\left[\mathrm{Me}_{6} \mathrm{TREN}\right]=200: 1:(\mathrm{x}):(\mathrm{y}):(\mathrm{x}+\mathrm{y}) ; 66 \% \quad(\mathrm{v} / \mathrm{v}) \quad \mathrm{MA}$ in DMSO, $T=25^{\circ} \mathrm{C}$, where $\mathrm{x} / \mathrm{y}=2.3$ or 0.8 and $\mathrm{x}+\mathrm{y}=1,3$, or 10 .

\begin{tabular}{|c|c|c|}
\hline Reaction & Rate Coefficient $\left.\mathbf{~ M}^{-\mathbf{1}} \mathbf{s}^{-1}\right)$ & Ref. \\
\hline $\mathrm{RX}+\mathrm{CuXL} \rightarrow \mathrm{R}+\mathrm{CuX} 2 \mathrm{~L}, k_{\mathrm{a} 1}$ & $2.0 \times 10^{2}$ & 1 \\
$\mathrm{PX}(\mathrm{s})+\mathrm{CuXL} \rightarrow \mathrm{P}(\mathrm{s})+\mathrm{CuX} 2 \mathrm{~L}, k_{\mathrm{a} 1}$ & $2.7 \times 10^{8}$ & 1 \\
\hline $\mathrm{R}+\mathrm{CuX} 2 \mathrm{~L} \rightarrow \mathrm{RX}+\mathrm{CuXL}, k_{\mathrm{d} 1}$ & $1.56 \times 10^{4}$ & 2 \\
$\mathrm{P}(\mathrm{s})+\mathrm{CuX} 2 \mathrm{~L} \rightarrow \mathrm{PX}(\mathrm{s})+\mathrm{CuXL}, k_{\mathrm{d} 1}$ & $1.6 \times 10^{4}$ & 3 \\
\hline $\mathrm{P}(\mathrm{s})+\mathrm{M} \rightarrow \mathrm{P}(\mathrm{s}+1), k_{\mathrm{p}}$ & $2.0 \times 10^{9}$ & 1 \\
\hline $\mathrm{R}+\mathrm{M} \rightarrow \mathrm{P}(1), k_{\mathrm{add}}$ & $1.0 \times 10^{8}$ & 1 \\
\hline $\mathrm{R}+\mathrm{R} \rightarrow \mathrm{RR}, k_{\mathrm{t} 0}$ & $1.0 \times 10^{8}$ & 1 \\
\hline $\mathrm{P}(\mathrm{s})+\mathrm{R} \rightarrow \mathrm{D}(\mathrm{s}), k_{\mathrm{tR}}$ & 0 & 4 \\
\hline $\mathrm{P}(\mathrm{s})+\mathrm{P}(\mathrm{r}) \rightarrow \mathrm{D}(\mathrm{s}+\mathrm{r}), k_{\mathrm{tc}}$ & & \\
\hline $\mathrm{P}(\mathrm{s})+\mathrm{P}(\mathrm{r}) \rightarrow \mathrm{Dd}(\mathrm{s}+\mathrm{r}), k_{\mathrm{td}}$ & &
\end{tabular}

Table S2. Kinetic model and rate coefficients for modeling of ICAR ATRP of methyl acrylate, under conditions: [MA]:[MBP]:[Cu $\left.{ }^{\mathrm{II}} \mathrm{Br}_{2}\right]:\left[\mathrm{Me}_{6} \mathrm{TREN}\right]:[\mathrm{AIBN}]=200: 1:(\mathrm{x}): 0.05:(\mathrm{y}) ; 66 \%$ (v/v) MA in DMSO, $T=25{ }^{\circ} \mathrm{C}$, where $\mathrm{x}=0.002$ or 0.006 and $\mathrm{y}=0.3$ or 0.05

\begin{tabular}{|c|c|c|}
\hline Reaction & Rate Coefficient $\left(\mathrm{M}^{-1} \mathrm{~s}^{-1}\right)$ & Ref. \\
\hline $\mathrm{I}_{2} \rightarrow \mathrm{I}+\mathrm{I}, k_{\mathrm{dc}}$ & $1.9 \times 10^{-5}$ & 5 \\
\hline $\begin{aligned} \mathrm{RX}+\mathrm{CuXL} & \rightarrow \mathrm{R}+\mathrm{CuX} 2 \mathrm{~L}, k_{\mathrm{a} 1} \\
\mathrm{PX}(\mathrm{s})+\mathrm{CuXL} & \rightarrow \mathrm{P}(\mathrm{s})+\mathrm{CuX} 2 \mathrm{~L}, k_{\mathrm{a} 1}\end{aligned}$ & $2.0 \times 10^{2}$ & 1 \\
\hline $\mathrm{IX}+\mathrm{CuXL} \rightarrow \mathrm{I}+\mathrm{CuX} 2 \mathrm{~L}, k_{\mathrm{a} 1 \mathrm{AIBN}}$ & $1.0 \times 10^{5}$ & scaled based on ref ${ }^{6}$ \\
\hline $\begin{aligned} \mathrm{R}+\mathrm{CuX} 2 \mathrm{~L} & \rightarrow \mathrm{RX}+\mathrm{CuXL}, k_{\mathrm{d} 1} \\
\mathrm{P}(\mathrm{s})+\mathrm{CuX} 2 \mathrm{~L} & \rightarrow \mathrm{PX}(\mathrm{s})+\mathrm{CuXL}, k_{\mathrm{d} 1}\end{aligned}$ & $2.7 \times 10^{8}$ & 1 \\
\hline $\mathrm{I}+\mathrm{CuX} 2 \mathrm{~L} \rightarrow \mathrm{IX}+\mathrm{CuXL}, k_{\mathrm{d} 1 \mathrm{AIBN}}$ & $5.0 \times 10^{7}$ & scaled based on ref ${ }^{6}$ \\
\hline $\mathrm{P}(\mathrm{s})+\mathrm{M} \rightarrow \mathrm{P}(\mathrm{s}+1), k_{\mathrm{p}}$ & $1.56 \times 10^{4}$ & 2 \\
\hline $\mathrm{R}+\mathrm{M} \rightarrow \mathrm{P}(1), k_{\mathrm{add}}$ & $1.6 \times 10^{4}$ & 3 \\
\hline $\mathrm{I}+\mathrm{M} \rightarrow \mathrm{P}(1), k_{\text {add AIBN }}$ & $3.7 \times 10^{2}$ & 7 \\
\hline $\mathrm{R}+\mathrm{R} \rightarrow \mathrm{RR}, k_{\mathrm{t} 0 \mathrm{RR}}$ & $2.0 \times 10^{9}$ & 1 \\
\hline $\mathrm{I}+\mathrm{R} \rightarrow \mathrm{IR}, k_{\mathrm{t} 0 \mathrm{IR}}$ & $1.3 \times 10^{9}$ & scaled based on refs ${ }^{1,8}$ \\
\hline $\mathrm{I}+\mathrm{I} \rightarrow \mathrm{II}, k_{\mathrm{t} 0 \mathrm{II}}$ & $8.0 \times 10^{8}$ & 8 \\
\hline $\mathrm{P}(\mathrm{s})+\mathrm{I} \rightarrow \mathrm{D}(\mathrm{s}), k_{\mathrm{tR}}$ & $1.0 \times 10^{8}$ & 1 \\
\hline $\mathrm{P}(\mathrm{s})+\mathrm{R} \rightarrow \mathrm{D}(\mathrm{s}), k_{\mathrm{tR}}$ & $1.0 \times 10^{8}$ & 1 \\
\hline $\mathrm{P}(\mathrm{s})+\mathrm{P}(\mathrm{r}) \rightarrow \mathrm{D}(\mathrm{s}+\mathrm{r}), k_{\mathrm{tc}}$ & $1.0 \times 10^{8}$ & 1 \\
\hline $\mathrm{P}(\mathrm{s})+\mathrm{P}(\mathrm{r}) \rightarrow \mathrm{Dd}(\mathrm{s}+\mathrm{r}), k_{\mathrm{td}}$ & 0 & 4 \\
\hline
\end{tabular}


Table S3. Kinetic model and rate coefficients for modeling of SARA ATRP of methyl acrylate, under conditions: [MA]:[MBP]:[Cu $\left.{ }^{\mathrm{II}} \mathrm{Br}_{2}\right]:\left[\mathrm{Me}_{6} \mathrm{TREN}\right]=200: 1: \mathrm{x}: 0.1 ; 66 \%(\mathrm{v} / \mathrm{v}) \mathrm{MA}$ in DMSO, $T=25{ }^{\circ} \mathrm{C}$, where $\mathrm{x}=0$ or 0.01 , with $l=10 \mathrm{~cm}$ of $d=0.25 \mathrm{~mm} \mathrm{Cu}^{0}$ wire $\left(\mathrm{S}=0.79 \mathrm{~cm}^{2}\right), V_{\text {total }}=10 \mathrm{~cm}^{3}$.

\begin{tabular}{|c|c|c|}
\hline Reaction & (apparent) Rate Coefficient & Ref. \\
\hline $\mathrm{CuXL}+\mathrm{CuXL} \rightarrow \mathrm{CuX} 2 \mathrm{~L}(+\mathrm{Cu}(0)), k_{\mathrm{disp}}$ & $\begin{array}{c}2.44 \times 10^{-7}\left(\mathrm{~s}^{-1}\right) \\
\left(3.1 \times 10^{-6} \mathrm{~cm} \mathrm{~s}^{-1} * 0.786 \mathrm{~cm}^{2} / 10 \mathrm{~cm}^{3}\right)\end{array}$ & 1 \\
\hline $\mathrm{CuX} 2 \mathrm{~L}(+\mathrm{Cu}(0)) \rightarrow \mathrm{CuXL}+\mathrm{CuXL}, k_{\mathrm{comp}}$ & $\begin{array}{c}2.75 \times 10^{-4}\left(\mathrm{~s}^{-1}\right) \\
\left(3.5 \times 10^{-3} \mathrm{~cm} \mathrm{~s}^{-1} * 0.786 \mathrm{~cm}^{2} / 10 \mathrm{~cm}^{3}\right)\end{array}$ & 1 \\
\hline $\begin{array}{c}\mathrm{RX}(+\mathrm{Cu}(0)) \rightarrow \mathrm{CuXL}+\mathrm{R}, k_{\mathrm{a} 0} \\
\mathrm{PX}(\mathrm{s})(+\mathrm{Cu}(0)) \rightarrow \mathrm{P}(\mathrm{s})+\mathrm{CuXL}, k_{\mathrm{a} 0}\end{array}$ & $\begin{array}{c}7.86 \times 10^{-6}\left(\mathrm{~s}^{-1}\right) \\
\left(1.0 \times 10^{-4} \mathrm{~cm} \mathrm{~s}^{-1} * 0.786 \mathrm{~cm}^{2} / 10 \mathrm{~cm}^{3}\right)\end{array}$ \\
\hline $\begin{array}{c}\mathrm{R}+\mathrm{CuXL} \rightarrow \mathrm{RX}(+\mathrm{Cu}(0)), k_{\mathrm{d} 0} \\
\mathrm{P}(\mathrm{s})+\mathrm{CuXL} \rightarrow \mathrm{PX}(\mathrm{s})(+\mathrm{Cu}(0)), k_{\mathrm{d} 0}\end{array}$ & \begin{tabular}{c}
$\left(1.2 \times 10^{-1} \mathrm{~cm} \mathrm{~s}^{-1} * 0.786 \mathrm{~cm}^{2} / 10 \mathrm{~cm}^{3}\right)$ \\
\hline $\mathrm{RX}+\mathrm{CuXL} \rightarrow \mathrm{R}+\mathrm{CuX} 2 \mathrm{~L}, k_{\mathrm{a} 1}$
\end{tabular} & 1 \\
\hline $\mathrm{PX}(\mathrm{s})+\mathrm{CuXL} \rightarrow \mathrm{P}(\mathrm{s})+\mathrm{CuX} 2 \mathrm{~L}, k_{\mathrm{a} 1}$ & $2.0 \times 10^{2}\left(\mathrm{M}^{-1} \mathrm{~s}^{-1}\right)$ & 1 \\
\hline $\begin{array}{c}\mathrm{R}+\mathrm{CuX} 2 \mathrm{~L} \rightarrow \mathrm{RX}+\mathrm{CuXL}, k_{\mathrm{d} 1} \\
\mathrm{P}(\mathrm{s})+\mathrm{CuX} 2 \mathrm{~L} \rightarrow \mathrm{PX}(\mathrm{s})+\mathrm{CuXL}, k_{\mathrm{d} 1}\end{array}$ & $2.7 \times 10^{8}\left(\mathrm{M}^{-1} \mathrm{~s}^{-1}\right)$ & 1 \\
\hline $\mathrm{P}(\mathrm{s})+\mathrm{M} \rightarrow \mathrm{P}(\mathrm{s}+1), k_{\mathrm{p}}$ & $1.56 \times 10^{4}\left(\mathrm{M}^{-1} \mathrm{~s}^{-1}\right)$ & 2 \\
\hline $\mathrm{R}+\mathrm{M} \rightarrow \mathrm{P}(1), k_{\mathrm{add}}$ & $1.6 \times 10^{4}\left(\mathrm{M}^{-1} \mathrm{~s}^{-1}\right)$ & 3 \\
\hline $\mathrm{R}+\mathrm{R} \rightarrow \mathrm{RR}, k_{\mathrm{t} 0}$ & $2.0 \times 10^{9}\left(\mathrm{M}^{-1} \mathrm{~s}^{-1}\right)$ & 1 \\
\hline $\mathrm{P}(\mathrm{s})+\mathrm{R} \rightarrow \mathrm{D}(\mathrm{s}), k_{\mathrm{tR}}$ & $1.0 \times 10^{8}\left(\mathrm{M}^{-1} \mathrm{~s}^{-1}\right)$ & 1 \\
\hline $\mathrm{P}(\mathrm{s})+\mathrm{P}(\mathrm{r}) \rightarrow \mathrm{D}(\mathrm{s}+\mathrm{r}), k_{\mathrm{tc}}$ & $1.0 \times 10^{8}\left(\mathrm{M}^{-1} \mathrm{~s}^{-1}\right)$ & 4 \\
\hline $\mathrm{P}(\mathrm{s})+\mathrm{P}(\mathrm{r}) \rightarrow \mathrm{Dd}(\mathrm{s}+\mathrm{r}), k_{\mathrm{td}}$ & $0\left(\mathrm{M}^{-1} \mathrm{~s}^{-1}\right)$ & \\
\hline
\end{tabular}

Table S4. Kinetic model and rate coefficients for modeling of SARA ATRP of methyl acrylate, under conditions: [MA]:[MBP]:[Cu $\left.{ }^{\mathrm{II}} \mathrm{Br}_{2}\right]:\left[\mathrm{Me}_{6} \mathrm{TREN}\right]=200: 1: \mathrm{x}: 0.1 ; 66 \%(\mathrm{v} / \mathrm{v}) \mathrm{MA}$ in DMSO, $T=25^{\circ} \mathrm{C}$, where $\mathrm{x}=0$ or 0.01 , with $l=1 \mathrm{~cm}$ of $d=0.25 \mathrm{~mm} \mathrm{Cu}^{0}$ wire $\left(\mathrm{S}=0.08 \mathrm{~cm}^{2}\right), V_{\text {total }}=10 \mathrm{~cm}^{3}$.

\begin{tabular}{|c|c|c|}
\hline Reaction & (apparent) Rate Coefficient & Ref. \\
\hline $\mathrm{CuXL}+\mathrm{CuXL} \rightarrow \mathrm{CuX} 2 \mathrm{~L}(+\mathrm{Cu}(0)), k_{\mathrm{disp}}$ & $\begin{array}{c}2.48 \times 10^{-8}\left(\mathrm{~s}^{-1}\right) \\
\left(3.1 \times 10^{-6} \mathrm{~cm} \mathrm{~s}^{-1} * 0.08 \mathrm{~cm}^{2} / 10 \mathrm{~cm}^{3}\right)\end{array}$ & 1 \\
\hline $\mathrm{CuX} 2 \mathrm{~L}(+\mathrm{Cu}(0)) \rightarrow \mathrm{CuXL}+\mathrm{CuXL}, k_{\mathrm{comp}}$ & $\begin{array}{c}2.8 \times 10^{-5}\left(\mathrm{~s}^{-1}\right) \\
\left(3.5 \times 10^{-3} \mathrm{~cm} \mathrm{~s}^{-1} * 0.08 \mathrm{~cm}^{2} / 10 \mathrm{~cm}^{3}\right)\end{array}$ & 1 \\
\hline $\begin{array}{c}\mathrm{RX}(+\mathrm{Cu}(0)) \rightarrow \mathrm{CuXL}+\mathrm{R}, k_{\mathrm{a} 0} \\
\mathrm{PX}(\mathrm{s})(+\mathrm{Cu}(0)) \rightarrow \mathrm{P}(\mathrm{s})+\mathrm{CuXL}, k_{\mathrm{a} 0}\end{array}$ & $\begin{array}{c}8.0 \times 10^{-7}\left(\mathrm{~s}^{-1}\right) \\
\left(1.0 \times 10^{-4} \mathrm{~cm} \mathrm{~s}^{-1} * 0.08 \mathrm{~cm}^{2} / 10 \mathrm{~cm}^{3}\right)\end{array}$ & 1 \\
\hline $\begin{array}{c}\mathrm{R}+\mathrm{CuXL} \rightarrow \mathrm{RX}(+\mathrm{Cu}(0)), k_{\mathrm{d} 0} \\
\mathrm{P}(\mathrm{s})+\mathrm{CuXL} \rightarrow \mathrm{PX}(\mathrm{s})(+\mathrm{Cu}(0)), k_{\mathrm{d} 0}\end{array}$ & $\begin{array}{c}9.6 \times 10^{-4}\left(\mathrm{~s}^{-1}\right) \\
\mathrm{RX}+\mathrm{CuXL} \rightarrow \mathrm{R}+\mathrm{CuX} 2 \mathrm{~L}, k_{\mathrm{a} 1}\end{array}$ & 1 \\
$\mathrm{PX}(\mathrm{s})+\mathrm{CuXL} \rightarrow \mathrm{P}(\mathrm{s})+\mathrm{CuX} 2 \mathrm{~L}, k_{\mathrm{a} 1}$ & $2.0 \times 10^{-1}\left(\mathrm{M}^{-1} \mathrm{~s}^{-1}\right)$ & 1 \\
\hline $\begin{array}{c}\mathrm{R}+\mathrm{CuX} 2 \mathrm{~L} \rightarrow \mathrm{RX}+\mathrm{CuXL}, k_{\mathrm{d} 1} \\
\mathrm{P}(\mathrm{s})+\mathrm{CuX} 2 \mathrm{~L} \rightarrow \mathrm{PX}(\mathrm{s})+\mathrm{CuXL}, k_{\mathrm{d} 1}\end{array}$ & $2.7 \times 10^{8}\left(\mathrm{M}^{-1} \mathrm{~s}^{-1}\right)$ & 1 \\
\hline
\end{tabular}




\begin{tabular}{|c|c|c|}
\hline $\mathrm{P}(\mathrm{s})+\mathrm{M} \rightarrow \mathrm{P}(\mathrm{s}+1), k_{\mathrm{p}}$ & $1.56 \times 10^{4}\left(\mathrm{M}^{-1} \mathrm{~s}^{-1}\right)$ & 2 \\
\hline $\mathrm{R}+\mathrm{M} \rightarrow \mathrm{P}(1), k_{\mathrm{add}}$ & $1.6 \times 10^{4}\left(\mathrm{M}^{-1} \mathrm{~s}^{-1}\right)$ & ${ }^{1}$ \\
\hline $\mathrm{R}+\mathrm{R} \rightarrow \mathrm{RR}, k_{\mathrm{t} 0}$ & $2.0 \times 10^{9}\left(\mathrm{M}^{-1} \mathrm{~s}^{-1}\right)$ & 1 \\
\hline $\mathrm{P}(\mathrm{s})+\mathrm{R} \rightarrow \mathrm{D}(\mathrm{s}), k_{\mathrm{tR}}$ & $1.0 \times 10^{8}\left(\mathrm{M}^{-1} \mathrm{~s}^{-1}\right)$ & 1 \\
\hline $\mathrm{P}(\mathrm{s})+\mathrm{P}(\mathrm{r}) \rightarrow \mathrm{D}(\mathrm{s}+\mathrm{r}), k_{\mathrm{tc}}$ & $1.0 \times 10^{8}\left(\mathrm{M}^{-1} \mathrm{~s}^{-1}\right)$ & 1 \\
\hline $\mathrm{P}(\mathrm{s})+\mathrm{P}(\mathrm{r}) \rightarrow \mathrm{Dd}(\mathrm{s}+\mathrm{r}), k_{\mathrm{td}}$ & $0\left(\mathrm{M}^{-1} \mathrm{~s}^{-1}\right)$ & 4 \\
\hline
\end{tabular}

Table S5. Kinetic model and rate coefficients for modeling of normal ATRP of methyl acrylate under conditions: $[\mathrm{MA}]:[\mathrm{EBiB}]:\left[\mathrm{Cu}^{\mathrm{I}} \mathrm{Br}\right]:\left[\mathrm{Cu}^{\mathrm{II}} \mathrm{Br}_{2}\right]:\left[\mathrm{Me}_{6} \mathrm{TREN}\right]=60: 1: 0.1: 0: 0.6 ; 50 \%(\mathrm{v} / \mathrm{v}) \mathrm{MA}$ in DMSO, $T=$ $25{ }^{\circ} \mathrm{C}$.

\begin{tabular}{|c|c|c|}
\hline Reaction & Rate Coefficient $\left(\mathrm{M}^{-1} \mathrm{~s}^{-1}\right)$ & Ref. \\
\hline $\begin{array}{l}\mathrm{RX}+\mathrm{CuXL} \rightarrow \mathrm{R}^{\cdot}+\mathrm{CuX} \mathrm{Cu}_{2} \mathrm{~L}, k_{\mathrm{a} 1} \\
\mathrm{R}^{\bullet}+\mathrm{CuX} \mathrm{Cu}_{2} \mathrm{~L} \rightarrow \mathrm{RX}+\mathrm{CuXL}, k_{\mathrm{d} 1}\end{array}$ & $\begin{array}{l}k_{\mathrm{a} 1}=1.63 \times 10^{3} \\
k_{\mathrm{d} 1}=4.22 \times 10^{7}\end{array}$ & scaled based on refs ${ }^{1,9}$ \\
\hline $\begin{array}{l}\mathrm{PX}(\mathrm{s})+\mathrm{CuXL} \rightarrow \mathrm{P}^{*}(\mathrm{~s})+\mathrm{CuX}_{2} \mathrm{~L}, k_{\mathrm{a} 1 \mathrm{p}} \\
\mathrm{P}^{*}(\mathrm{~s})+\mathrm{CuX} \mathrm{X}_{2} \mathrm{~L} \rightarrow \mathrm{PX}(\mathrm{s})+\mathrm{CuXL}, k_{\mathrm{d} 1 \mathrm{p}}\end{array}$ & $\begin{array}{l}k_{\mathrm{alp}}=2.0 \times 10^{2} \\
k_{\mathrm{dlp}}=2.7 \times 10^{8}\end{array}$ & 1 \\
\hline $\mathrm{P}^{*}(\mathrm{~s})+\mathrm{M} \rightarrow \mathrm{P}^{*}(\mathrm{~s}+1), k_{\mathrm{p}}$ & $1.56 \times 10^{4}$ & 2 \\
\hline $\mathrm{R}^{\bullet}+\mathrm{M} \rightarrow \mathrm{P}^{\bullet}(1), k_{\mathrm{add}}$ & $1.2 \times 10^{3}$ & 7 \\
\hline $\mathrm{R}^{\cdot}+\mathrm{R}^{\cdot} \rightarrow \mathrm{RR}, k_{\mathrm{t} 0}$ & $3.0 \times 10^{8}$ & this work \\
\hline $\mathrm{P}^{\circ}(\mathrm{s})+\mathrm{R}^{\cdot} \rightarrow \mathrm{D}(\mathrm{s}), k_{\mathrm{tR}}$ & $1.0 \times 10^{8}$ & 1 \\
\hline $\mathrm{P}^{\circ}(\mathrm{s})+\mathrm{P}^{\circ}(\mathrm{r}) \rightarrow \mathrm{D}(\mathrm{s}+\mathrm{r}), k_{\mathrm{tc}}$ & $1.0 \times 10^{8}$ & 1 \\
\hline $\mathrm{P}^{*}(\mathrm{~s})+\mathrm{P}^{*}(\mathrm{r}) \rightarrow \mathrm{DH}(\mathrm{s})+\mathrm{D}^{=}(\mathrm{r}), k_{\mathrm{td}}$ & 0 & 4 \\
\hline
\end{tabular}

Table S6. Kinetic model and rate coefficients for modeling of SARA ATRP of methyl acrylate in the presence of $\mathrm{Cu}^{0}$ wire under conditions: $[\mathrm{MA}]:[\mathrm{EBiB}]:\left[\mathrm{Cu}{ }^{\mathrm{I}} \mathrm{Br}\right]:\left[\mathrm{Cu}^{\mathrm{II}} \mathrm{Br}_{2}\right]:\left[\mathrm{Me}_{6} \mathrm{TREN}\right]=60: 1: 0: 0: 0.12$; $50 \%(\mathrm{v} / \mathrm{v}) \mathrm{MA}$ in DMSO, $T=25^{\circ} \mathrm{C}, \mathrm{Cu}^{0}$ wire: $l=5 \mathrm{~cm}, d=0.25 \mathrm{~mm}\left(S=0.39 \mathrm{~cm}^{2}\right), V_{\text {total }}=4 \mathrm{~cm}^{3}$.

\begin{tabular}{|c|c|c|}
\hline Reaction & (apparent) Rate Coefficient & Ref. \\
\hline $\mathrm{CuXL}+\mathrm{CuXL} \rightarrow \mathrm{CuX}_{2} \mathrm{~L}(+\mathrm{Cu}(0)), k_{\mathrm{disp}}$ & $\begin{array}{c}3.05 \times 10^{-7}\left(\mathrm{~s}^{-1}\right) \\
\left(3.1 \times 10^{-6} \mathrm{~cm} \mathrm{~s}^{-1} 0.394 \mathrm{~cm}^{2} / 4.0 \mathrm{~cm}^{3}\right)\end{array}$ & 1 \\
\hline $\mathrm{CuX}_{2} \mathrm{~L}(+\mathrm{Cu}(0)) \rightarrow \mathrm{CuXL}+\mathrm{CuXL}, k_{\mathrm{comp}}$ & $\begin{array}{c}3.45 \times 10^{-4}\left(\mathrm{~s}^{-1}\right) \\
\left(3.5 \times 10^{-3} \mathrm{~cm} \mathrm{~s}^{-1} * 0.394 \mathrm{~cm}^{2} / 4.0 \mathrm{~cm}^{3}\right)\end{array}$ & 1 \\
\hline $\mathrm{RX}(+\mathrm{Cu}(0)) \rightarrow \mathrm{CuXL}+\mathrm{R}^{\cdot}, k_{\mathrm{a} 0}$ & $\begin{array}{c}6.6 \times 10^{-5}\left(\mathrm{~s}^{-1}\right) \\
\left(6.7 \times 10^{-4} \mathrm{~cm} \mathrm{~s}^{-1} * 0.394 \mathrm{~cm}^{2} / 4.0 \mathrm{~cm}^{3}\right)\end{array}$ & $\begin{array}{c}\text { scaled based } \\
\text { on refs }\end{array}$ \\
\hline $\mathrm{R}^{\cdot}+\mathrm{CuXL} \rightarrow \mathrm{RX}(+\mathrm{Cu}(0)), k_{\mathrm{d} 0}$ & $\begin{array}{c}1.5 \times 10^{-3}\left(\mathrm{~s}^{-1}\right) \\
\left(1.5 \times 10^{-2} \mathrm{~cm} \mathrm{~s}^{-1} * 0.394 \mathrm{~cm}^{2} / 4.0 \mathrm{~cm}^{3}\right)\end{array}$ & 1 \\
\hline $\mathrm{PX}(\mathrm{s})(+\mathrm{Cu}(0)) \rightarrow \mathrm{P}^{\cdot}(\mathrm{s})+\mathrm{CuXL}, k_{\mathrm{a} 0 \mathrm{p}}$ & $\begin{array}{c}9.85 \times 10^{-6}\left(\mathrm{~s}^{-1}\right) \\
\left(1.0 \times 10^{-4} \mathrm{~cm} \mathrm{~s}^{-1} * 0.394 \mathrm{~cm}^{2} / 4.0 \mathrm{~cm}^{3}\right)\end{array}$ & 1 \\
\hline $\mathrm{P}^{\cdot}(\mathrm{s})+\mathrm{CuXL} \rightarrow \mathrm{PX}(\mathrm{s})(+\mathrm{Cu}(0)), k_{\mathrm{d} 0 \mathrm{p}}$ & $\begin{array}{c}1.2 \times 10^{-2}\left(\mathrm{~s}^{-1}\right) \\
\left(1.2 \times 10^{-1} \mathrm{~cm} \mathrm{~s}^{-1} * 0.394 \mathrm{~cm}^{2} / 4.0 \mathrm{~cm}^{3}\right)\end{array}$ & 1 \\
\hline
\end{tabular}




\begin{tabular}{|c|c|c|}
\hline $\begin{array}{l}\mathrm{RX}+\mathrm{CuXL} \rightarrow \mathrm{R}^{\cdot}+\mathrm{CuX} \mathrm{C}_{2} \mathrm{~L}, k_{\mathrm{a} 1} \\
\mathrm{R}^{\cdot}+\mathrm{CuX} \mathrm{Cu}_{2} \mathrm{~L} \rightarrow \mathrm{RX}+\mathrm{CuXL}, k_{\mathrm{d} 1}\end{array}$ & $\begin{aligned} k_{\mathrm{a} 1} & =1.63 \times 10^{3}\left(\mathrm{M}^{-1} \mathrm{~s}^{-1}\right) \\
k_{\mathrm{d} 1} & =4.22 \times 10^{7}\left(\mathrm{M}^{-1} \mathrm{~s}^{-1}\right)\end{aligned}$ & $\begin{array}{c}\text { scaled based } \\
\text { on refs } 1,9\end{array}$ \\
\hline $\begin{array}{l}\mathrm{PX}(\mathrm{s})+\mathrm{CuXL} \rightarrow \mathrm{P}^{*}(\mathrm{~s})+\mathrm{CuX} \mathrm{Cu}_{2} \mathrm{~L}, k_{\mathrm{a} 1 \mathrm{p}} \\
\mathrm{P}^{*}(\mathrm{~s})+\mathrm{CuX} \mathrm{Cu}_{2} \mathrm{PX}(\mathrm{s})+\mathrm{CuXL}, k_{\mathrm{d} 1 \mathrm{p}}\end{array}$ & $\begin{array}{l}k_{\mathrm{alp}}=2.0 \times 10^{2}\left(\mathrm{M}^{-1} \mathrm{~s}^{-1}\right) \\
k_{\mathrm{d} 1 \mathrm{p}}=2.7 \times 10^{8}\left(\mathrm{M}^{-1} \mathrm{~s}^{-1}\right)\end{array}$ & 1 \\
\hline $\mathrm{P}^{*}(\mathrm{~s})+\mathrm{M} \rightarrow \mathrm{P}^{*}(\mathrm{~s}+1), k_{\mathrm{p}}$ & $1.56 \times 10^{4}\left(\mathrm{M}^{-1} \mathrm{~s}^{-1}\right)$ & 2 \\
\hline $\mathrm{R}^{\cdot}+\mathrm{M} \rightarrow \mathrm{P}^{\circ}(1), k_{\text {add }}$ & $1.2 \times 10^{3}\left(\mathrm{M}^{-1} \mathrm{~s}^{-1}\right)$ & ( \\
\hline $\mathrm{R}^{\cdot}+\mathrm{R}^{\cdot} \rightarrow \mathrm{RR}, k_{\mathrm{t} 0}$ & $3.0 \times 10^{8}\left(\mathrm{M}^{-1} \mathrm{~s}^{-1}\right)$ & this work \\
\hline $\mathrm{P}^{*}(\mathrm{~s})+\mathrm{R}^{\cdot} \rightarrow \mathrm{D}(\mathrm{s}), k_{\mathrm{tR}}$ & $1.0 \times 10^{8}\left(\mathrm{M}^{-1} \mathrm{~s}^{-1}\right)$ & 1 \\
\hline $\mathrm{P}^{\bullet}(\mathrm{s})+\mathrm{P}^{\cdot}(\mathrm{r}) \rightarrow \mathrm{D}(\mathrm{s}+\mathrm{r}), k_{\mathrm{tc}}$ & $1.0 \times 10^{8}\left(\mathrm{M}^{-1} \mathrm{~s}^{-1}\right)$ & 1 \\
\hline $\mathrm{P}^{*}(\mathrm{~s})+\mathrm{P}^{*}(\mathrm{r}) \rightarrow \mathrm{DH}(\mathrm{s})+\mathrm{D}^{=}(\mathrm{r}), k_{\mathrm{td}}$ & $0\left(\mathrm{M}^{-1} \mathrm{~s}^{-1}\right)$ & 4 \\
\hline
\end{tabular}

Table S7. Kinetic model and rate coefficients for modeling of normal ATRP of methyl acrylate under conditions $[\mathrm{MA}]:[\mathrm{MBP}]:\left[\mathrm{Cu}^{\mathrm{I}} \mathrm{Br}\right]:\left[\mathrm{Cu}^{\mathrm{II}} \mathrm{Br}_{2}\right]:\left[\mathrm{Me}_{6} \mathrm{TREN}\right]=60: 1: 0.1: 0: 0.6 ; 50 \%(\mathrm{v} / \mathrm{v}) \mathrm{MA}$ in DMSO, $T=$ $25{ }^{\circ} \mathrm{C}$.

\begin{tabular}{|c|c|c|}
\hline Reaction & Rate Coefficient $\left(\mathrm{M}^{-1} \mathrm{~s}^{-1}\right)$ & Ref. \\
\hline $\begin{array}{c}\mathrm{RX}+\mathrm{CuXL} \rightarrow \mathrm{R}^{\cdot}+\mathrm{CuX}_{2} \mathrm{~L}, k_{\mathrm{a} 1} \\
\mathrm{R}^{\cdot}+\mathrm{CuX} \mathrm{X}_{2} \mathrm{~L} \rightarrow \mathrm{RX}+\mathrm{CuXL}, k_{\mathrm{d} 1} \\
\mathrm{PX}(\mathrm{s})+\mathrm{CuXL} \rightarrow \mathrm{P}^{*}(\mathrm{~s})+\mathrm{CuX}_{2} \mathrm{~L}, k_{\mathrm{a} 1} \\
\mathrm{P}^{*}(\mathrm{~s})+\mathrm{CuX} \mathrm{L} \mathrm{L} \rightarrow \mathrm{PX}(\mathrm{s})+\mathrm{CuXL}, k_{\mathrm{d} 1}\end{array}$ & $\begin{aligned} k_{\mathrm{a} 1} & =2.0 \times 10^{2} \\
k_{\mathrm{d} 1} & =2.7 \times 10^{8}\end{aligned}$ & 1 \\
\hline $\mathrm{P}^{\circ}(\mathrm{s})+\mathrm{M} \rightarrow \mathrm{P}^{\circ}(\mathrm{s}+1), k_{\mathrm{p}}$ & $1.56 \times 10^{4}$ & 2 \\
\hline $\mathrm{R}^{\bullet}+\mathrm{M} \rightarrow \mathrm{P}^{\bullet}(1), k_{\text {add }}$ & $1.6 \times 10^{4}$ & 3 \\
\hline $\mathrm{R}^{\bullet}+\mathrm{R}^{\bullet} \rightarrow \mathrm{RR}, k_{\mathrm{t} 0}$ & $2.0 \times 10^{9}$ & 1 \\
\hline $\mathrm{P}^{*}(\mathrm{~s})+\mathrm{R}^{\cdot} \rightarrow \mathrm{D}(\mathrm{s}), k_{\mathrm{tR}}$ & $1.0 \times 10^{8}$ & 1 \\
\hline $\mathrm{P}^{\circ}(\mathrm{s})+\mathrm{P}^{\circ}(\mathrm{r}) \rightarrow \mathrm{D}(\mathrm{s}+\mathrm{r}), k_{\mathrm{tc}}$ & $1.0 \times 10^{8}$ & 1 \\
\hline $\mathrm{P}^{*}(\mathrm{~s})+\mathrm{P}^{*}(\mathrm{r}) \rightarrow \mathrm{DH}(\mathrm{s})+\mathrm{D}^{=}(\mathrm{r}), k_{\mathrm{td}}$ & 0 & 4 \\
\hline
\end{tabular}


Table S8. Kinetic model and rate coefficients for modeling of SARA ATRP of methyl acrylate in the presence of $\mathrm{Cu}^{0}$ wire under conditions: [MA]:[MBP]:[Cu $\left.{ }^{\mathrm{I}} \mathrm{Br}\right]:\left[\mathrm{Cu}^{\mathrm{II}} \mathrm{Br}_{2}\right]:\left[\mathrm{Me}_{6} \mathrm{TREN}\right]=60: 1: 0: 0: 0.12$; $50 \%(\mathrm{v} / \mathrm{v}) \mathrm{MA}$ in DMSO, $T=25^{\circ} \mathrm{C}, \mathrm{Cu}^{0}$ wire: $l=5 \mathrm{~cm}, d=0.25 \mathrm{~mm}\left(S=0.39 \mathrm{~cm}^{2}\right), V_{\text {total }}=4 \mathrm{~cm}^{3}$

\begin{tabular}{|c|c|c|}
\hline Reaction & (apparent) Rate Coefficient & Ref. \\
\hline $\mathrm{CuXL}+\mathrm{CuXL} \rightarrow \mathrm{CuX}_{2} \mathrm{~L}(+\mathrm{Cu}(0)), k_{\mathrm{disp}}$ & $\begin{array}{c}3.05 \times 10^{-7}\left(\mathrm{~s}^{-1}\right) \\
\left(3.1 \times 10^{-6} \mathrm{~cm} \mathrm{~s}^{-1} * 0.394 \mathrm{~cm}^{2} / 4.0 \mathrm{~cm}^{3}\right)\end{array}$ & 1 \\
\hline $\mathrm{CuX}_{2} \mathrm{~L}(+\mathrm{Cu}(0)) \rightarrow \mathrm{CuXL}+\mathrm{CuXL}, k_{\mathrm{comp}}$ & $\begin{array}{c}3.45 \times 10^{-4}\left(\mathrm{~s}^{-1}\right) \\
\left(3.5 \times 10^{-3} \mathrm{~cm} \mathrm{~s}^{-1} * 0.394 \mathrm{~cm}^{2} / 4.0 \mathrm{~cm}^{3}\right)\end{array}$ & 1 \\
\hline $\begin{aligned} \mathrm{RX}(+\mathrm{Cu}(0)) & \rightarrow \mathrm{CuXL}+\mathrm{R}^{\circ}, k_{\mathrm{a} 0} \\
\mathrm{PX}(\mathrm{s})(+\mathrm{Cu}(0)) & \rightarrow \mathrm{P}^{\circ}(\mathrm{s})+\mathrm{CuXL}, k_{\mathrm{a} 0}\end{aligned}$ & $\begin{array}{c}9.85 \times 10^{-6}\left(\mathrm{~s}^{-1}\right) \\
\left(1.0 \times 10^{-4} \mathrm{~cm} \mathrm{~s}^{-1} * 0.394 \mathrm{~cm}^{2} / 4.0 \mathrm{~cm}^{3}\right)\end{array}$ & 1 \\
\hline $\begin{aligned} \mathrm{R}^{\cdot}+\mathrm{CuXL} & \rightarrow \mathrm{RX}(+\mathrm{Cu}(0)), k_{\mathrm{d} 0} \\
\mathrm{P}^{*}(\mathrm{~s})+\mathrm{CuXL} & \rightarrow \mathrm{PX}(\mathrm{s})(+\mathrm{Cu}(0)), k_{\mathrm{d} 0}\end{aligned}$ & $\begin{array}{c}1.2 \times 10^{-2}\left(\mathrm{~s}^{-1}\right) \\
\left(1.2 \times 10^{-1} \mathrm{~cm} \mathrm{~s}^{-1} * 0.394 \mathrm{~cm}^{2} / 4.0 \mathrm{~cm}^{3}\right)\end{array}$ & 1 \\
\hline $\begin{array}{c}\mathrm{RX}+\mathrm{CuXL} \rightarrow \mathrm{R}^{\circ}+\mathrm{CuX}_{2} \mathrm{~L}, k_{\mathrm{a} 1} \\
\mathrm{R}^{\circ}+\mathrm{CuX}_{2} \mathrm{~L} \rightarrow \mathrm{RX}+\mathrm{CuXL}, k_{\mathrm{d} 1} \\
\mathrm{PX}(\mathrm{s})+\mathrm{CuXL} \rightarrow \mathrm{P}^{\circ}(\mathrm{s})+\mathrm{CuX}_{2} \mathrm{~L}, k_{\mathrm{a} 1} \\
\mathrm{P}^{\circ}(\mathrm{s})+\mathrm{CuX}_{2} \mathrm{~L} \rightarrow \mathrm{PX}(\mathrm{s})+\mathrm{CuXL}, k_{\mathrm{d} 1}\end{array}$ & $\begin{array}{l}k_{\mathrm{a} 1}=2.0 \times 10^{2}\left(\mathrm{M}^{-1} \mathrm{~s}^{-1}\right) \\
k_{\mathrm{d} 1}=2.7 \times 10^{8}\left(\mathrm{M}^{-1} \mathrm{~s}^{-1}\right)\end{array}$ & 1 \\
\hline $\mathrm{P}^{\circ}(\mathrm{s})+\mathrm{M} \rightarrow \mathrm{P}^{\circ}(\mathrm{s}+1), k_{\mathrm{p}}$ & $1.56 \times 10^{4}\left(\mathrm{M}^{-1} \mathrm{~s}^{-1}\right)$ & 2 \\
\hline $\mathrm{R}^{\bullet}+\mathrm{M} \rightarrow \mathrm{P}^{\bullet}(1), k_{\mathrm{add}}$ & $1.6 \times 10^{4}\left(\mathrm{M}^{-1} \mathrm{~s}^{-1}\right)$ & 3 \\
\hline $\mathrm{R}^{\cdot}+\mathrm{R}^{\cdot} \rightarrow \mathrm{RR}, k_{\mathrm{t} 0}$ & $2.0 \times 10^{9}\left(\mathrm{M}^{-1} \mathrm{~s}^{-1}\right)$ & 1 \\
\hline $\mathrm{P}^{\circ}(\mathrm{s})+\mathrm{R}^{\cdot} \rightarrow \mathrm{D}(\mathrm{s}), k_{\mathrm{tR}}$ & $1.0 \times 10^{8}\left(\mathrm{M}^{-1} \mathrm{~s}^{-1}\right)$ & 1 \\
\hline $\mathrm{P}^{\bullet}(\mathrm{s})+\mathrm{P}^{\bullet}(\mathrm{r}) \rightarrow \mathrm{D}(\mathrm{s}+\mathrm{r}), k_{\mathrm{tc}}$ & $1.0 \times 10^{8}\left(\mathrm{M}^{-1} \mathrm{~s}^{-1}\right)$ & 1 \\
\hline $\mathrm{P}^{\bullet}(\mathrm{s})+\mathrm{P}^{\bullet}(\mathrm{r}) \rightarrow \mathrm{DH}(\mathrm{s})+\mathrm{D}^{=}(\mathrm{r}), k_{\mathrm{td}}$ & $0\left(\mathrm{M}^{-1} \mathrm{~s}^{-1}\right)$ & \\
\hline
\end{tabular}

Table S9. Kinetic model and rate coefficients for modeling of normal ATRP of methyl acrylate with varied $k_{\mathrm{a} 1}$ values, under conditions: $[\mathrm{MA}]:[\mathrm{RX}]:\left[\mathrm{Cu}^{\mathrm{I}} \mathrm{Br}\right]:\left[\mathrm{Cu}^{\mathrm{II}} \mathrm{Br}_{2}\right]:[\mathrm{L}]=200: 1: 0.09: 0.01: 0.1 ; 50 \%(\mathrm{v} / \mathrm{v})$ MA in DMSO, $T=25^{\circ} \mathrm{C}$.

\begin{tabular}{|c|c|c|}
\hline Reaction & Rate Coefficient $\left(\mathrm{M}^{-1} \mathrm{~s}^{-1}\right)$ & Ref. \\
\hline $\begin{array}{c}\mathrm{RX}+\mathrm{CuXL} \rightarrow \mathrm{R}^{\cdot}+\mathrm{CuX}_{2} \mathrm{~L}, k_{\mathrm{a} 1} \\
\mathrm{R}^{\circ}+\mathrm{CuX} 2 \mathrm{~L} \rightarrow \mathrm{RX}+\mathrm{CuXL}, k_{\mathrm{d} 1} \\
\mathrm{PX}(\mathrm{s})+\mathrm{CuXL} \rightarrow \mathrm{P}^{\circ}(\mathrm{s})+\mathrm{CuX}_{2} \mathrm{~L}, k_{\mathrm{a} 1} \\
\mathrm{P}^{\circ}(\mathrm{s})+\mathrm{CuX} 2 \mathrm{~L} \rightarrow \mathrm{PX}(\mathrm{s})+\mathrm{CuXL}, k_{\mathrm{d} 1}\end{array}$ & $\begin{array}{c}k_{\mathrm{a} 1}=1 \times 10^{-2}-1 \times 10^{4} \\
k_{\mathrm{d} 1}=1 \times 10^{7}\end{array}$ & - \\
\hline $\mathrm{P}^{*}(\mathrm{~s})+\mathrm{M} \rightarrow \mathrm{P}^{*}(\mathrm{~s}+1), k_{\mathrm{p}}$ & $1.56 \times 10^{4}$ & 2 \\
\hline $\mathrm{R}^{\circ}+\mathrm{M} \rightarrow \mathrm{P}^{\circ}(1), k_{\text {add }}$ & $1.6 \times 10^{4}$ & 3 \\
\hline $\mathrm{R}^{\bullet}+\mathrm{R}^{\circ} \rightarrow \mathrm{RR}, k_{\mathrm{t} 0}$ & $2.0 \times 10^{9}$ & 1 \\
\hline $\mathrm{P}^{*}(\mathrm{~s})+\mathrm{R}^{\cdot} \rightarrow \mathrm{D}(\mathrm{s}), k_{\mathrm{tR}}$ & $1.0 \times 10^{8}$ & 1 \\
\hline $\mathrm{P}^{*}(\mathrm{~s})+\mathrm{P}^{*}(\mathrm{r}) \rightarrow \mathrm{D}(\mathrm{s}+\mathrm{r}), k_{\mathrm{tc}}$ & $1.0 \times 10^{8}$ & 1 \\
\hline $\mathrm{P}^{*}(\mathrm{~s})+\mathrm{P}^{*}(\mathrm{r}) \rightarrow \mathrm{DH}(\mathrm{s})+\mathrm{D}^{=}(\mathrm{r}), k_{\mathrm{td}}$ & 0 & 4 \\
\hline
\end{tabular}



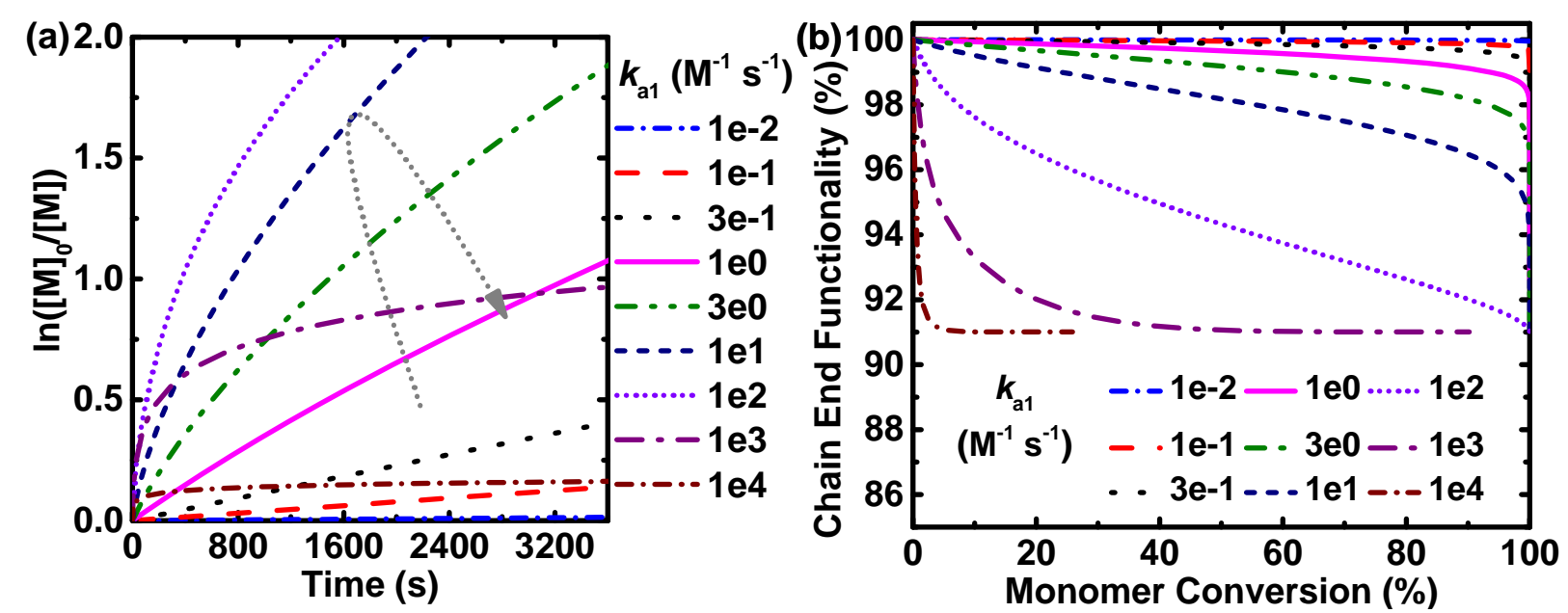

Figure S1 Simulations of normal ATRP of methyl acrylate with varied $k_{\mathrm{a} 1}$ values, under conditions [MA]:[RX]:[Cu $\left.{ }^{\mathrm{I}} \mathrm{Br}\right]:\left[\mathrm{Cu}^{\mathrm{II}} \mathrm{Br}_{2}\right]:[\mathrm{L}]=200: 1: 0.09: 0.01: 0.1 ; 50 \%(\mathrm{v} / \mathrm{v}) \mathrm{MA}$ in DMSO, $T=25^{\circ} \mathrm{C}$. Reactions and rate coefficients used for PREDICI simulations are presented in Table S9 a) semi-logarithmic kinetic plot and $\mathrm{b}$ ) chain end functionality calculated as $\mathrm{CEF}=1-T_{\mathrm{mol} \%}$, where $T_{\mathrm{mol} \%}=\left([\mathrm{R}-\mathrm{X}]_{0}-[\mathrm{R}-\mathrm{X}]_{\mathrm{t}}\right.$ $\left.-[\mathrm{P}-\mathrm{X}]_{\mathrm{t}}\right) /[\mathrm{R}-\mathrm{X}]_{0}$ (Note: Maximum \% of terminated chains is $9 \%$, as $[\mathrm{RX}]:\left[\mathrm{Cu} \mathrm{u}^{\mathrm{I}} \mathrm{Br}\right]=1: 0.09$ ). 
Kinetic simulations of ICAR ATRP with various ligands

Rate of polymerization in ICAR ATRP depends on the rate of radical generation from thermal initiator (e.g. AIBN), cf. Equation 9 in main text. This rate does not depend on the choice of ligand $\left(K_{\mathrm{ATRP}}\right)$. However, $\left[\mathrm{Cu}^{\mathrm{I}}\right] /\left[\mathrm{Cu}^{\mathrm{II}}\right]$ ratio adjusts based on $\left[\mathrm{R}^{\circ}\right]$ to fit $K_{\mathrm{ATRP}}$. Therefore, choice of ligand in ICAR ATRP determines the relative concentrations of $\left[\mathrm{Cu}^{\mathrm{I}}\right]$ and $\left[\mathrm{Cu}^{\mathrm{II}}\right]$ (i.e. the $\left[\mathrm{Cu}^{\mathrm{I}}\right] /\left[\mathrm{Cu}^{\mathrm{II}}\right]$ ratio). Since MWD depends on $\left[\mathrm{Cu}^{\mathrm{II}}\right]$ (cf. Equation 12 in main text), ligand choice is critical for obtaining polymers with narrow molecular weight distribution.
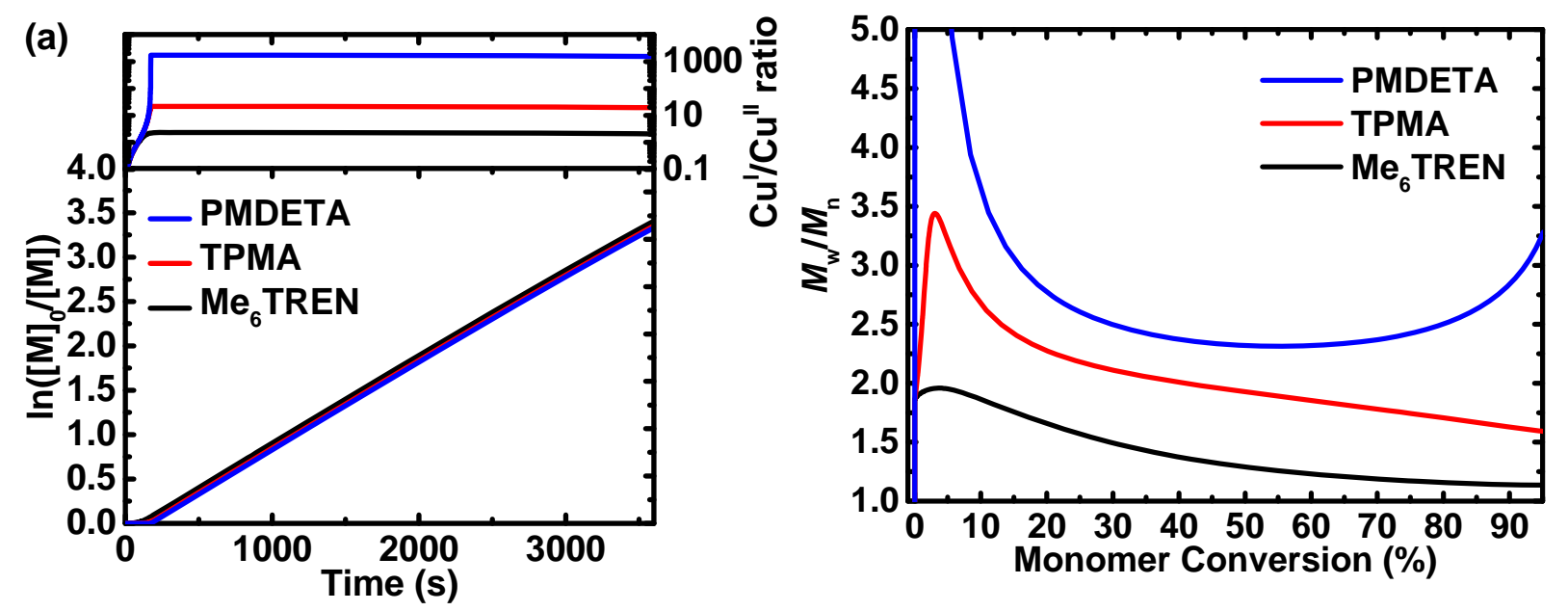

Figure S2. (a) Kinetics of ICAR ATRP and evolution of $\left[\mathrm{Cu}^{\mathrm{I}}\right] /\left[\mathrm{Cu}^{\mathrm{II}}\right]$ ratio, and (b) $M_{\mathrm{w}} / M_{\mathrm{n}}$ as a function of monomer conversion. Conditions: [MA]:[MBP]:[C $\left.{ }^{\mathrm{II}} \mathrm{Br}_{2}\right]:[\mathrm{L}]:[\mathrm{AIBN}]=200: 1: 0.002: 0.05: 0.3 ; 66 \%$ $(\mathrm{v} / \mathrm{v}) \mathrm{MA}$ in DMSO, $T=25^{\circ} \mathrm{C}$. Reactions and rate coefficients used for PREDICI simulations are presented in Table S2, where $k_{\mathrm{a} 1}=2.7 \times 10^{1} \mathrm{M}^{-1} \mathrm{~s}^{-1}$ (TPMA) or $1.2 \mathrm{M}^{-1} \mathrm{~s}^{-1}$ (PMDETA) and $k_{\mathrm{d} 1}=3.4 \times 10^{8} \mathrm{M}^{-1} \mathrm{~s}^{-1}$ (TPMA) or $1.2 \times 10^{9} \mathrm{M}^{-1} \mathrm{~s}^{-1}$ (PMDETA) (rate coefficients scaled based on ref. $\left.{ }^{1,9}\right)$. 

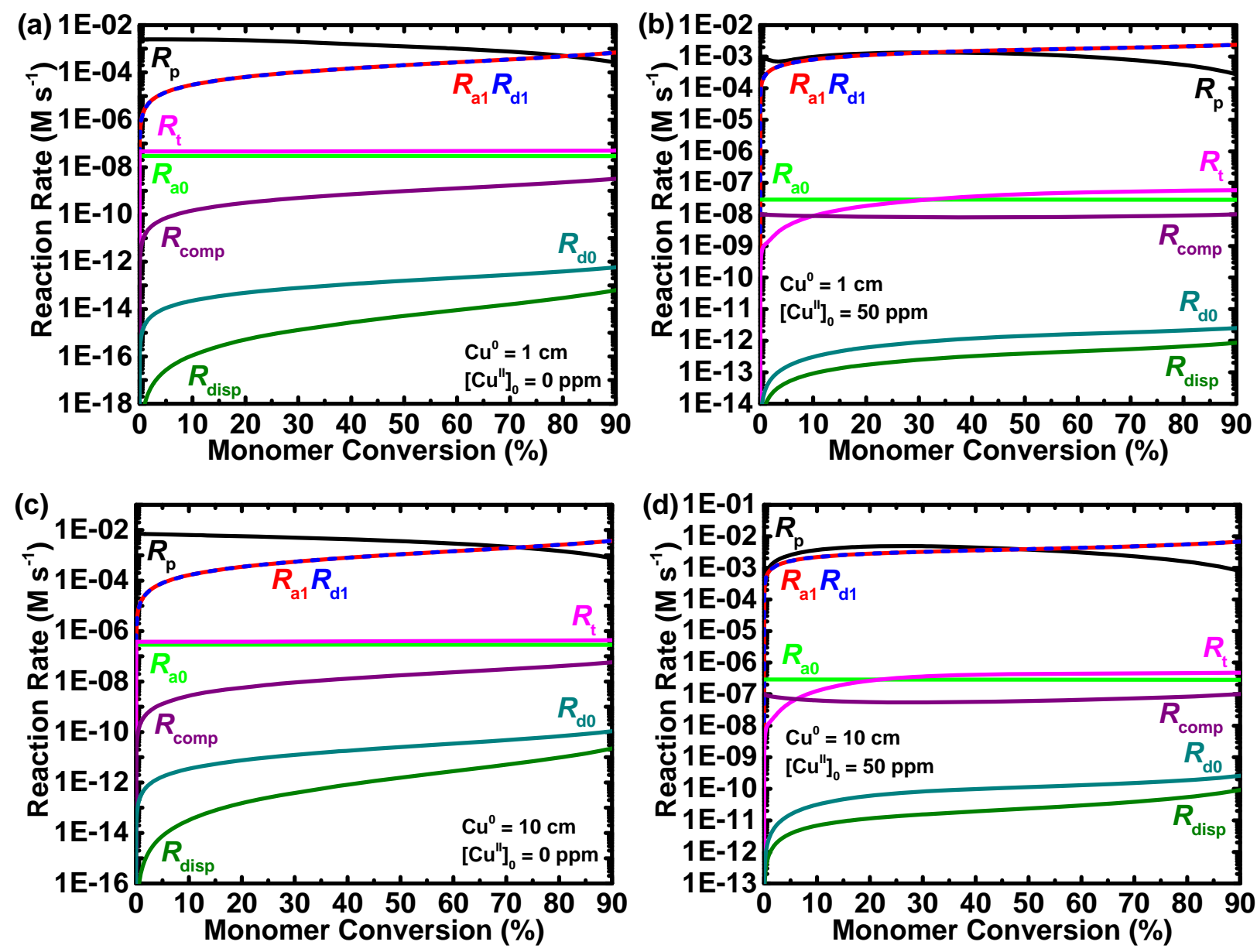

Figure S3. Rates of propagation $\left(R_{\mathrm{p}}\right)$, activation by $\mathrm{Cu}^{\mathrm{I}}\left(R_{\mathrm{a} 1}\right)$, deactivation by $\mathrm{Cu}^{\mathrm{II}}\left(R_{\mathrm{d} 1}\right)$, termination $\left(R_{\mathrm{t}}\right)$, activation by $\mathrm{Cu}^{0}\left(R_{\mathrm{a} 0}\right)$, comproportionation $\left(R_{\text {comp }}\right)$, deactivation by $\mathrm{Cu}^{\mathrm{I}}\left(R_{\mathrm{d} 0}\right)$ and disproportionation $\left(R_{\text {disp }}\right)$ as a function of monomer conversion. Conditions: [MA]:[MBP]: $\left[\mathrm{Cu}^{\mathrm{II}} \mathrm{Br}_{2}\right]:\left[\mathrm{Me}_{6} \mathrm{TREN}\right]=200: 1: \mathrm{x}: 0.1 ; 66 \%(\mathrm{v} / \mathrm{v}) \mathrm{MA}$ in DMSO, $\mathrm{T}=25^{\circ} \mathrm{C}$, where $\mathrm{x}=0(\mathrm{a}$, c) or $0.01(\mathrm{~b}, \mathrm{~d})$, with $l=1 \mathrm{~cm} \mathrm{(a,b)} \mathrm{or} l=10 \mathrm{~cm}(\mathrm{c}, \mathrm{d})$ of $d=0.25 \mathrm{~mm} \mathrm{Cu}{ }^{0}$ wire $\left(S=0.08 \mathrm{~cm}^{2}\right.$ or 0.79 $\left.\mathrm{cm}^{2}\right), V_{\text {total }}=10 \mathrm{~cm}^{3}$. Reactions and rate coefficients used for PREDICI simulations are presented in Tables S3 and S4. 


\section{Simulations of Normal ATRP and SARA ATRP with MBP as initiator}

Choice of alkyl halide is critically important. While highly reactive in activation by $\mathrm{Cu} / \mathrm{L},{ }^{9} \mathrm{EBiB}$ produces a tertiary radical which adds the first MA monomer slower $\left(k_{\text {add }}=1.2 \times 10^{3} \mathrm{M}^{-1} \mathrm{~s}^{-1}\right)^{7}$ than the subsequent MA propagation $\left(k_{\mathrm{p}}=1.56 \times 10^{4} \mathrm{M}^{-1} \mathrm{~s}^{-1}\right){ }^{2}$ Fast activation together with slow first monomer addition leads to high concentration of initiating radicals and, in consequence, to the fast termination and slower polymerization. When MBP was used for ATRP under conditions analogous to those from ref. ${ }^{11}$, $60 \%$ monomer conversion was reached in $1 \mathrm{~h}$ (Figure S7, or $40 \%$ conversion in $5 \mathrm{~min}$ !), as compared to $2 \mathrm{~h}$ when EBiB was used. ${ }^{11}$ Addition of the monomer to the secondary radical, produced by MBP $\left(k_{\mathrm{add}}=\right.$ $\left.1.6 \times 10^{4} \mathrm{M}^{-1} \mathrm{~s}^{-1}\right),{ }^{3}$ is comparable to the MA propagation, ${ }^{2}$ providing efficient consumption of the initiator and more uniform growth of the polymer chains.
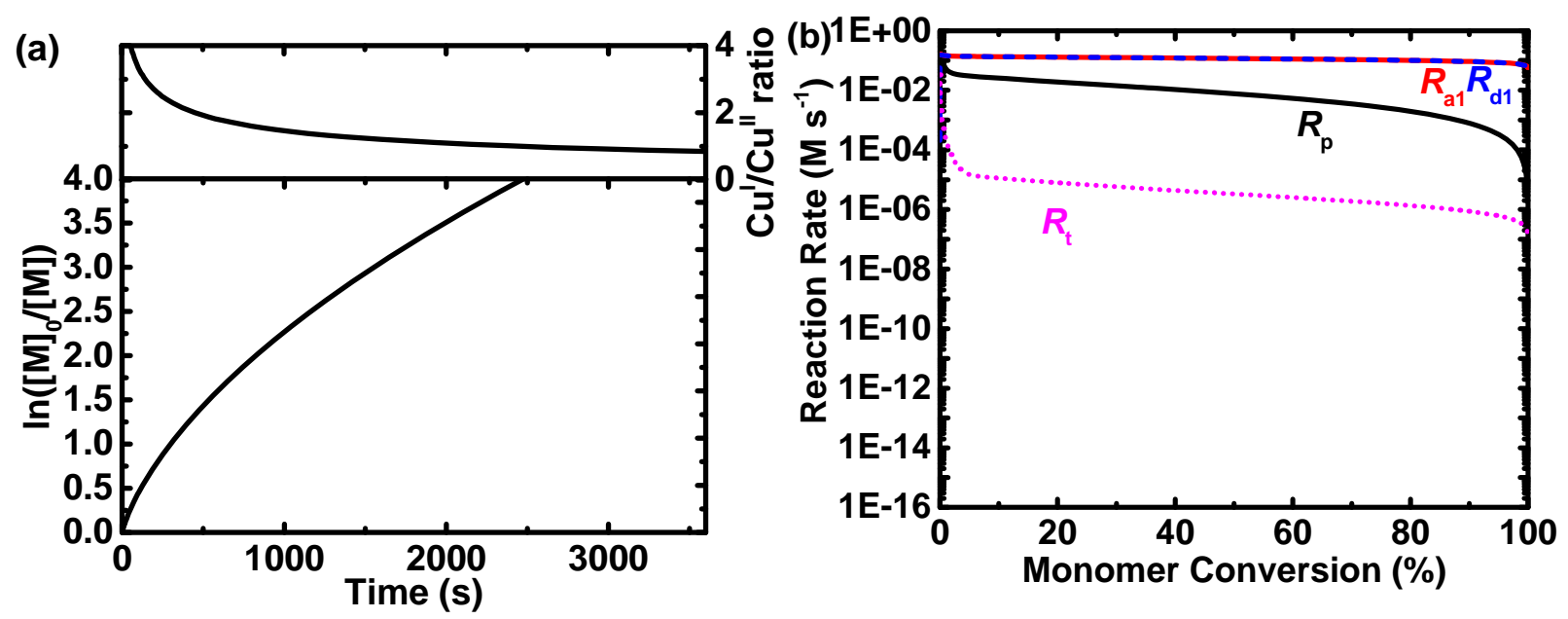

Figure S4. (a) Kinetics of normal ATRP and evolution of $\left[\mathrm{Cu}^{\mathrm{I}}\right] /\left[\mathrm{Cu}^{\mathrm{II}}\right]$ ratio, and $(\mathrm{b})$ rates of respective reactions as a function of monomer conversion. Conditions: [MA]:[MBP]:[C $\left.{ }^{\mathrm{I}} \mathrm{Br}\right]:\left[\mathrm{Cu}^{\mathrm{II}} \mathrm{Br}_{2}\right]:\left[\mathrm{Me}_{6} \mathrm{TREN}\right]$ $=60: 1: 0.1: 0: 0.6 ; 50 \%(\mathrm{v} / \mathrm{v}) \mathrm{MA}$ in DMSO, $T=25^{\circ} \mathrm{C}$. Reactions and rate coefficients used for PREDICI simulations are presented in Table S7. 

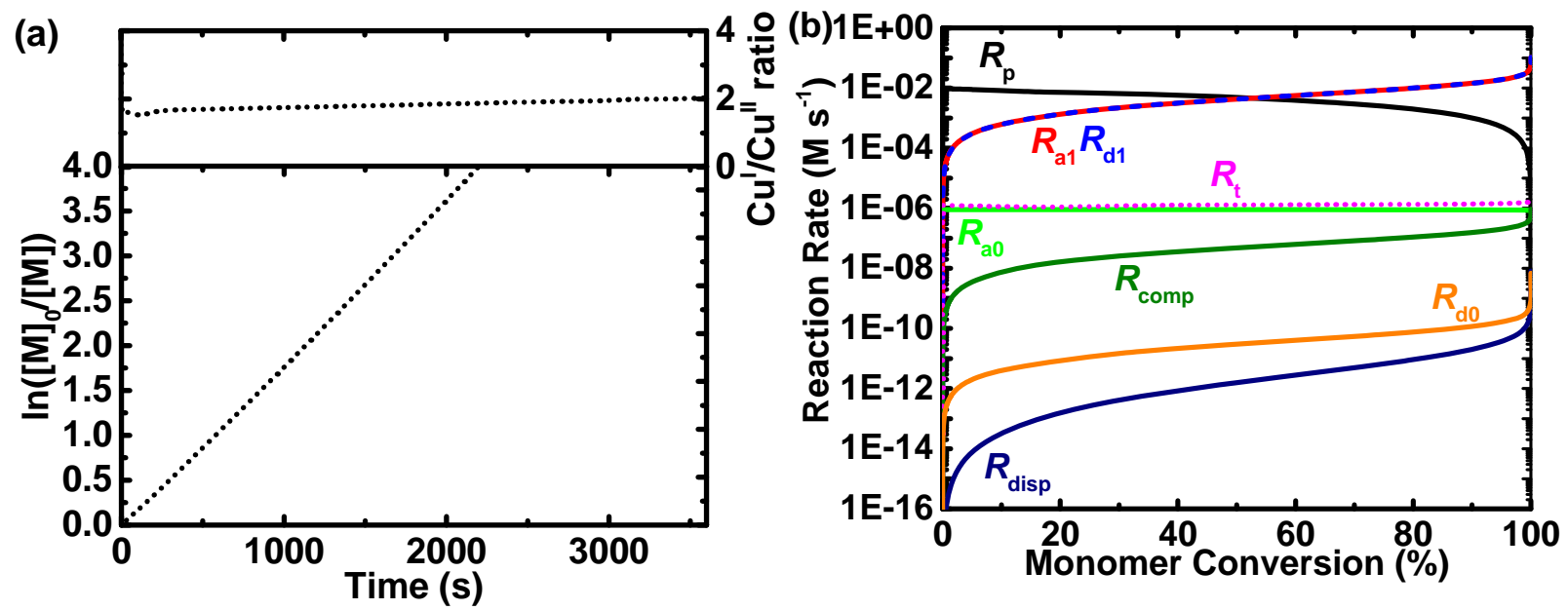

Figure S5. a) Kinetics of SARA ATRP and evolution of $\left[\mathrm{Cu}^{\mathrm{I}}\right] /\left[\mathrm{Cu}^{\mathrm{II}}\right]$ ratio, and (b) rates of respective reactions as a function of monomer conversion. Conditions: [MA]:[MBP]:[Cu $\left.{ }^{\mathrm{I}} \mathrm{Br}\right]:\left[\mathrm{Cu}^{\mathrm{II}} \mathrm{Br}_{2}\right]:\left[\mathrm{Me}_{6} \mathrm{TREN}\right]$ $=60: 1: 0: 0: 0.12 ; 50 \%(\mathrm{v} / \mathrm{v}) \mathrm{MA}$ in DMSO, $T=25{ }^{\circ} \mathrm{C}, \mathrm{Cu}^{0}$ wire: $l=5 \mathrm{~cm} d=0.25 \mathrm{~mm}\left(S=0.39 \mathrm{~cm}^{2}\right)$, $V_{\text {total }}=4 \mathrm{~cm}^{3}$. Reactions and rate coefficients used for PREDICI simulations are presented in Table S8.
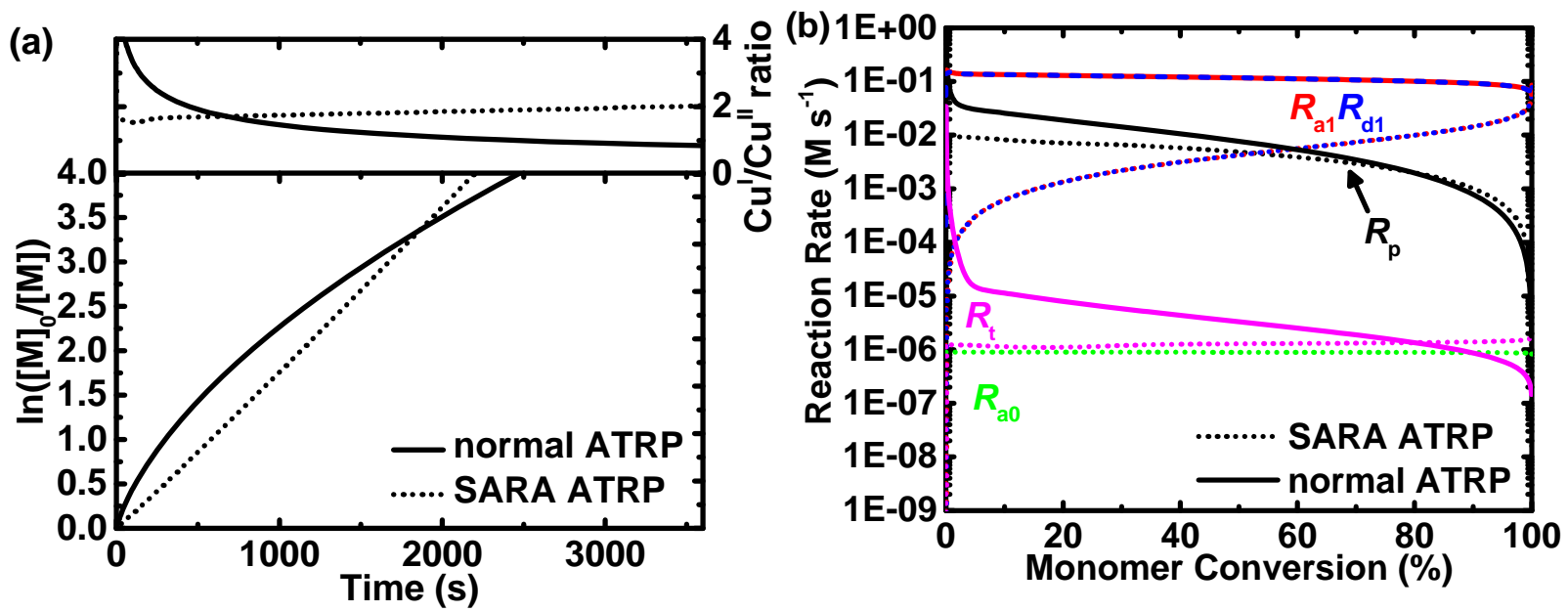

Figure S6. (a) Kinetics of Normal ATRP and SARA ATRP and evolution of $\left[\mathrm{Cu}^{\mathrm{I}}\right] /\left[\mathrm{Cu}^{\mathrm{II}}\right]$ ratio, and (b) rates of propagation, activation, deactivation, termination and $\mathrm{Cu}^{0}$ activation as a function of monomer conversion. Conditions: Normal ATRP $[\mathrm{MA}]:[\mathrm{MBP}]:\left[\mathrm{Cu}{ }^{\mathrm{I}} \mathrm{Br}\right]:\left[\mathrm{Cu}^{\mathrm{II}} \mathrm{Br}_{2}\right]:\left[\mathrm{Me}_{6} \mathrm{TREN}\right]=60: 1: 0.1: 0: 0.6$; SARA ATRP [MA]:[MBP]:[Cu $\left.{ }^{\mathrm{I}} \mathrm{Br}\right]:\left[\mathrm{Cu}^{\mathrm{II}} \mathrm{Br}_{2}\right]:\left[\mathrm{Me}_{6} \mathrm{TREN}\right]=60: 1: 0: 0: 0.12 ; \mathrm{Cu}^{0}$ wire: $l=5 \mathrm{~cm}, d=0.25$ $\mathrm{mm}\left(S=0.39 \mathrm{~cm}^{2}\right), V_{\text {total }}=4 \mathrm{~cm}^{3}$. All reactions $50 \%(\mathrm{v} / \mathrm{v}) \mathrm{MA}$ in DMSO, $T=25{ }^{\circ} \mathrm{C}$. Reactions and rate coefficients used for PREDICI simulations are presented in Tables S7 and S8. 


\section{$\underline{\text { Induction period in SARA ATRP with EBiB }}$}

Longer induction periods in SARA ATRP with $\mathrm{EBiB}$ initiator, reported in literature ${ }^{12}$ may be related to several plausibly contributing factors. They include passivation of $\mathrm{Cu}^{0}$ surface by oxides or high MW polymer formed at early stages of the reaction, slow addition of primary radicals to monomer and high rate coefficients of termination of primary radicals. Indeed, when MBP was used (faster addition to monomer) no induction period was observed. Diminished induction periods in the presence of initially added $\mathrm{Cu}^{\mathrm{II}}$ species suggest larger contribution of comproportionation, proceeding by OSET, rather than direct activation via ISET. It should also be noted that relatively exothermic polymerization of acrylates can lead to autoacceleration, especially in the absence of initially added deactivators. Detailed kinetic studies of SARA ATRP with EBiB are currently under investigation.

\section{Normal ATRP of methyl acrylate using MBP as initiator}

A Schlenk flask was equipped with magnetic stirrer and evacuated and backfilled with $\mathrm{N}_{2}$ four times. DMSO $(2 \mathrm{~mL})$ and methyl acrylate $(2 \mathrm{~mL})$ were added with a $\mathrm{N}_{2}$ purged syringes. $\mathrm{Me}_{6}$ TREN (59 $\mu \mathrm{L}$; $0.22 \mathrm{mmol})$ and MBP $(41 \mu \mathrm{L} ; 0.37 \mathrm{mmol})$ were injected with microsyringes. Finally, $\mathrm{Cu}^{\mathrm{I}} \mathrm{Br}(5 \mathrm{mg}$; $0.037 \mathrm{mmol}$ ) was carefully added under positive $\mathrm{N}_{2}$ pressure. The flask was sealed and the reaction mixture was allowed to polymerize at $25{ }^{\circ} \mathrm{C}$. Samples were withdrawn periodically for ${ }^{1} \mathrm{H}$ NMR and GPC analysis. The results are presented in Figure S7.
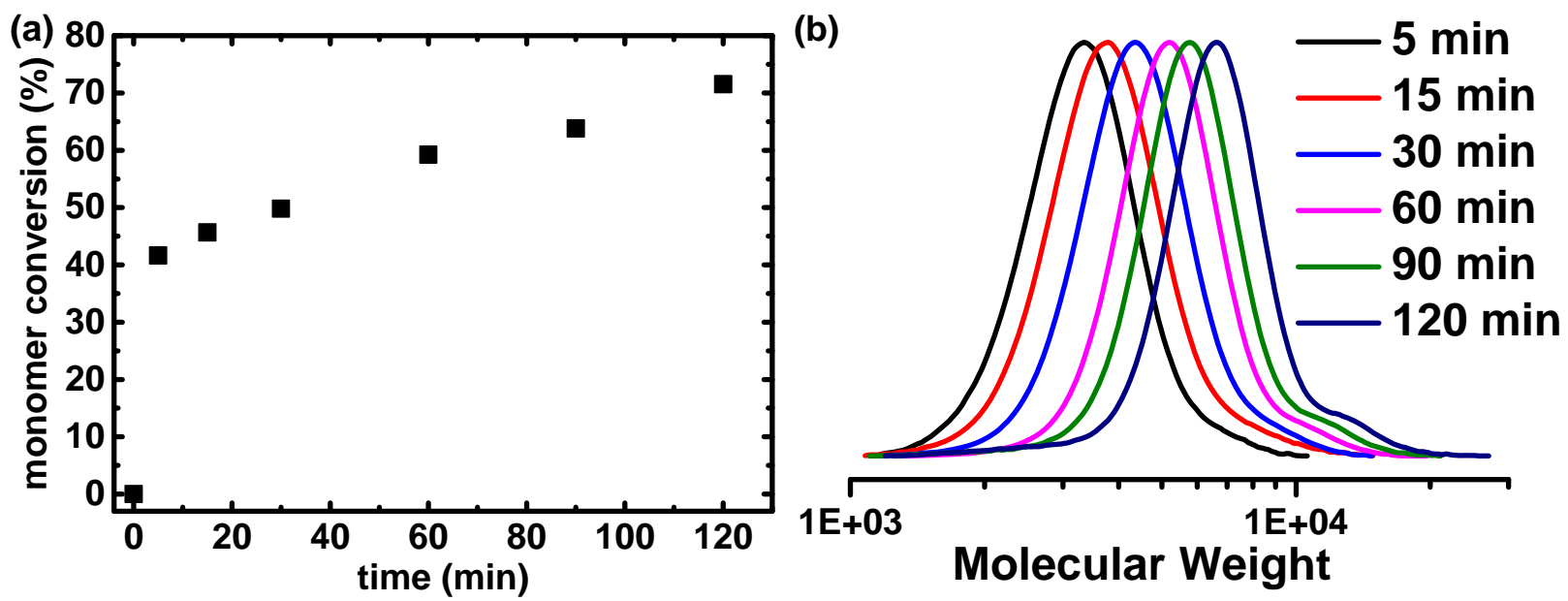

Figure S7. Normal ATRP of methyl acrylate under conditions: [MA]:[MBP]:[Cu $\left.{ }^{\mathrm{I} B r}\right]:\left[\mathrm{Cu}^{\mathrm{II}} \mathrm{Br}_{2}\right]:\left[\mathrm{Me}_{6} \mathrm{TREN}\right]=60: 1: 0.1: 0: 0.6 ; 50 \%$ (v/v) MA in DMSO, $T=22{ }^{\circ} \mathrm{C}$. a) monomer conversion $v s$. time, and $b$ ) evolution of molecular weight with time. 


\section{Evaluation of small molecule termination rate coefficient $\left(k_{\mathrm{t} 0}\right)$}

The value of termination rate coefficient $\left(k_{\mathrm{t} 0}\right)$ for methyl isobutyrate radicals derived from methyl $\alpha$-bromoisobutyrate (MBiB) in DMSO was calculated based on ref. ${ }^{8}$ to be $8 \times 10^{8} \mathrm{M}^{-1} \mathrm{~s}^{-1}$. For $\mathrm{EBiB}$ derived radical, due to the presence of ethyl group, instead of methyl, termination rate coefficient might be lower. Additionally, the value should reflect the increasing viscosity of the reaction mixture, due to polymerization, which will slow down termination. Finally, due to the addition of $\mathrm{Cu}^{\mathrm{I}} \mathrm{Br}$ as the last component in the experimental procedure, it is plausible that dissolution and complexation of $\mathrm{Cu}^{\mathrm{I}} \mathrm{Br}$ solid to form $\mathrm{Cu}^{\mathrm{I}} \mathrm{Br} / \mathrm{L}$ takes some time. This would decrease initial concentration of activator and therefore reduce the amount of radicals produced at the beginning of reaction, additionally diminishing initial termination. Therefore, taking all these aspects into consideration, $k_{\mathrm{t} 0}$ was approximated as $3 \times$ $10^{8} \mathrm{M}^{-1} \mathrm{~s}^{-1}$. The effect of $k_{\mathrm{t} 0}$ value on polymerization of MA in normal ATRP and SARA ATRP is presented in Figure S8. The effect of termination rate coefficient is much stronger on normal ATRP due to much faster activation of alkyl halides by $\mathrm{Cu}^{\mathrm{I}}$.

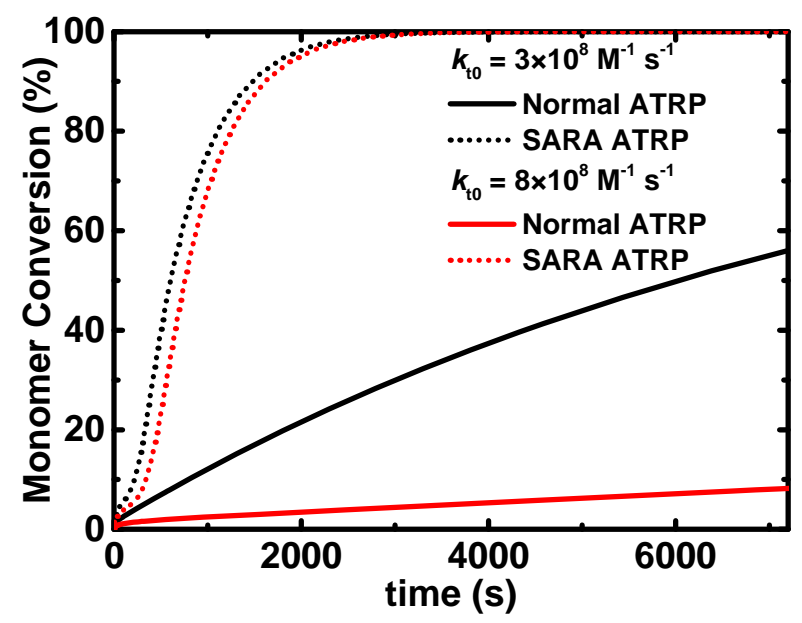

Figure S8. Simulations of normal ATRP and SARA ATRP of methyl acrylate in the presence of $\mathrm{Cu}^{0}$ wire with varied $k_{\mathrm{t} 0}$ value, under conditions: Normal ATRP [MA]:[EBiB]:[Cu $\left.{ }^{\mathrm{I}} \mathrm{Br}\right]:\left[\mathrm{Cu}^{\mathrm{II}} \mathrm{Br}_{2}\right]:\left[\mathrm{Me}_{6} \mathrm{TREN}\right]$ $=60: 1: 0.1: 0: 0.6 ; 50 \% \quad(\mathrm{v} / \mathrm{v}) \quad \mathrm{MA}$ in DMSO, $T=25{ }^{\circ} \mathrm{C} ; \quad$ SARA ATRP [MA]:[EBiB]:[Cu $\left.{ }^{\mathrm{I}} \mathrm{Br}\right]:\left[\mathrm{Cu}^{\mathrm{II}} \mathrm{Br}_{2}\right]:\left[\mathrm{Me}_{6} \mathrm{TREN}\right]=60: 1: 0: 0: 0.12 ; 50 \%(\mathrm{v} / \mathrm{v}) \mathrm{MA}$ in DMSO, $T=25{ }^{\circ} \mathrm{C}, \mathrm{Cu}^{0}$ wire: $l=5 \mathrm{~cm}, d=0.25 \mathrm{~mm}\left(S=0.39 \mathrm{~cm}^{2}\right), V_{\text {total }}=4 \mathrm{~cm}^{3}$. Reactions and rate coefficients used for PREDICI simulations are presented in Tables S5 and S6, with $k_{\mathrm{t} 0}=3 \times 10^{8}$ or $8 \times 10^{8}\left(\mathrm{M}^{-1} \mathrm{~s}^{-1}\right)$. 


\section{References}

(1) Zhong, M.; Wang, Y.; Krys, P.; Konkolewicz, D.; Matyjaszewski, K., Reversible-Deactivation Radical

Polymerization in the Presence of Metallic Copper. Kinetic Simulation. Macromolecules 2013, 46, 3816-3827.

(2) Buback, M.; Kurz, C. H.; Schmaltz, C., Pressure dependence of propagation rate coefficients in free-radical homopolymerizations of methyl acrylate and dodecyl acrylate. Macromol. Chem. Phys. 1998, 199, 1721-1727.

(3) Knühl, B.; Marque, S.; Fischer, H., Absolute Rate Constants for the Addition of the 1-(tertButoxy)carbonylethyl Radical to Alkenes in Solution. Helv. Chim. Acta 2001, 84, 2290-2300.

(4) Moad, G.; Solomon, D. H., The chemistry of free radical polymerization. 2nd Ed. ed.; Elsevier: Amsterdam, 2006.

(5) Brandrup, J.; Immergut, E. H.; Grulke, E. A.; Abe, A.; Bloch, D. R., Polymer handbook. Wiley New York: 1999; Vol. 89.

(6) Balili, M. N. C.; Pintauer, T., Kinetic Studies of the Initiation Step in Copper Catalyzed Atom Transfer Radical Addition (ATRA) in the Presence of Free Radical Diazo Initiators as Reducing Agents. Inorg. Chem. 2010, 49, 5642-5649.

(7) Fischer, H.; Radom, L., Factors Controlling the Addition of Carbon-Centered Radicals to Alkenes-An Experimental and Theoretical Perspective. Angew. Chem. Int. Ed. 2001, 40, 1340-1371.

(8) Braunecker, W. A.; Tsarevsky, N. V.; Gennaro, A.; Matyjaszewski, K., Thermodynamic Components of the Atom Transfer Radical Polymerization Equilibrium: Quantifying Solvent Effects. Macromolecules 2009, 42, 6348-6360.

(9) Tang, W.; Kwak, Y.; Braunecker, W.; Tsarevsky, N. V.; Coote, M. L.; Matyjaszewski, K., Understanding Atom Transfer Radical Polymerization: Effect of Ligand and Initiator Structures on the Equilibrium Constants. $J$. Am. Chem. Soc. 2008, 130, 10702-10713.

(10) Ribelli, T. G.; Krys, P.; Cong, Y.; Matyjaszewski, K., Model Studies of Alkyl Halide Activation and Comproportionation Relevant to RDRP in the Presence of Cu0. Macromolecules 2015, 48, 8428-8436.

(11) Alsubaie, F.; Anastasaki, A.; Nikolaou, V.; Simula, A.; Nurumbetov, G.; Wilson, P.; Kempe, K.; Haddleton, D. M., Investigating the Mechanism of Copper(0)-Mediated Living Radical Polymerization in Organic Media. Macromolecules 2015, 48, 5517-5525.

(12) Gao, Y.; Zhao, T.; Zhou, D.; Greiser, U.; Wang, W., Insights into relevant mechanistic aspects about the induction period of $\mathrm{Cu} 0 / \mathrm{Me} 6 \mathrm{TREN}-$ mediated reversible-deactivation radical polymerization. Chem. Commun. 2015, 51, 14435-14438. 